\title{
Las tecnologías socioculturales en los procesos de innovación de los migrantes de amenidad y por estilos de vida. El caso del destino turístico de Pucón, Chile ${ }^{1}$
}

\author{
Adriana María Otero², Hugo Marcelo Zunino ${ }^{3}$ y Mariana Rodríguez ${ }^{4}$
}

\begin{abstract}
RESUMEN
Muchos destinos de montaña reciben el aporte de migrantes de diversas culturas que generan innovaciones. En general las investigaciones se centran en analizar las tecnologías sociales presentes en los procesos de innovación desde la perspectiva del desarrollo económico, dejando de lado los beneficios sociales y culturales que ellas implican para sus comunidades. Este trabajo apunta a conocer las tecnologías socioculturales de los migrantes por estilo de vida en la ciudad de Pucón e indagar en qué medida ellas, contribuyen a un proceso de innovación sustentado en el intercambio cultural entre los migrantes y los habitantes de las comunidades receptoras. Para ello desplegamos un abordaje metodológico cualitativo e interpretativo sustentado en 12 entrevistas en profundidad realizadas a migrantes que recientemente se han asentado en la localidad y que han iniciado procesos de innovación. Argumentamos que las tecnologías socioculturales de los migrantes por estilos de vida de Pucón están basadas en cambiar a las personas que cambian las cosas, para así comprender las formas que asumen los procesos de innovación.
\end{abstract}

Palabras clave: Tecnologías socioculturales, migrantes de amenidad, migrantes por estilo de vida, Pucón.

\begin{abstract}
Many mountain destinations receive the contribution of migrants from diverse cultures that generate enterprises and innovations. In general the literature has focused on analyzing social technologies present in process of innovation from the perspective of economic development, leaving aside the social and cultural benefits they entail for their communities. The objective of this paper is to understand the sociocultural technologies of amenity and lifestyle migrants in Pucón and investigate to what extent they contribute to a sustained process of innovation in cultural exchanges between migrants and the inhabitants of the host communities. We deploy a qualitative and exploratory methodology based on 12 in-depth interviews conducted with 12 individuals recently settled in the area and that have initiate processes of innovation. We argue that the sociocultural technologies deployed by the lifestyle migrants show the relevance of considering the contextual mode to comprehend the forms that the process of innovation acquires.
\end{abstract}

Keywords: Sociocultural technologies, amenity migration, lifestyle migration, Pucón.

Este artículo es un resultado del proyecto MEC 800140080 y FONDECYT 1150944 del Comisión Nacional de Investigación Científica y Tecnológica de Chile. Los autores agradecen a CONICYT el financiamiento otorgado que hicieron posible realizar este trabajo. Artículo recibido el 1 de abril de 2016, aceptado el 5 de diciembre de 2016 y corregido el 17 de abril de 2017.

CEPLADES-Turismo, Facultad de Turismo. Universidad Nacional del Comahue (Argentina). E-mail: oteroadrianamaria@gmail.com

Núcleo de Ciencias Sociales y Humanidades, Departamento de Ciencias Sociales, Universidad de La Frontera (Chile).

E-mail: hugo.zunino@ufrontera.cl

4. CEPLADES-Turismo, Facultad de Turismo. Universidad Nacional del Comahue (Argentina). E-mail: marianfatu@hotmail.com 
En los últimos años muchos lugares de montaña que son destinos turísticos han recibido el aporte de nuevos habitantes de diversas culturas, transformándose en elementos de innovación e emprendimiento (Zunino et al., 2016). Los estudios que interpretan los procesos de movilidad, incluyen como facilitadores de la migración de personas de grandes ciudades a localidades intermedias y pequeñas, la mayor accesibilidad, las oportunidades profesionales y para el desarrollo de emprendimientos, y la posibilidad de entornos de vida más tranquilos para sus familias (Otero y González, 2014).

Desde la perspectiva de la migración por estilo de vida estos desplazamientos son de carácter intencionado y están dirigidos a cambiar las condiciones de vida en base las aspiraciones y sueños que forjan en el lugar de destino del movimiento (Benson, 2011; Benson \& O'Reilly, 2009). Las investigaciones que se han realizado en el Sur de Chile han detectado un flujo importante de migrantes hacia centros turísticos y pueblos cordilleranos impactando en forma poblacional de lugares como Malalcahuello, Puerto Varas y Villarrica (Zunino et al., 2013). En particular, en la Ciudad de Pucón algunos estudios han enfatizado la contribución que realizan los migrantes nacionales y extranjeros en dinamizar la economía y vivificar la cultura local (Zunino e Hidalgo, 2010; Zebryte et al., 2015), mientras que otros han alertado sobre el carácter regresivo que imprime al desarrollo local, debido al incremento de la segregación social y aumento de la especulación inmobiliaria (Zunino et al., 2011)

Si bien esta forma de movilidad puede generar consecuencias no previstas sobre las localidades que en muchos casos son necesarias de regular, se ha observado, por ejemplo, que en San Carlos de Bariloche, uno de los destinos turísticos más importantes del Norte de la Patagonia Argentina, que estos flujos de personas han abierto espacios para la realización de subjetividades más progresistas y cosmopolitas. La migración constante en esta ciudad desde fines del siglo XIX ha constituido la base de nuevas formaciones sociales y culturales, a partir de sujetos más reflexivos y, por tanto, más críticos, de sus propias condiciones sociales de existencia. A partir de su capital cultural internalizado en forma de conocimiento y habilidades y su capital experiencial generan una diversidad de innovaciones socioculturales en las comunidades que los reciben.

Dada la creatividad y la voluntad de emprendimiento que acarrean muchos migrantes, los convierte en potencial fuente de innovación, es decir en promotores de modificaciones en las prácticas individuales y sociales (hábitos, trayectorias, costumbres) y en la trama simbólico-significativa en las cuales se insertan (Margulis et al., 2014). El estudio de la innovación a nivel territorial cobra relevancia para comprender los procesos sociales, culturales y económicos que inducen y repercuten sobre el desarrollo local. En general las investigaciones se centran fundamentalmente en analizar estas tecnologías desde la perspectiva del desarrollo económico, dejando de lado un análisis sistemático del amplio espectro de beneficios sociales y culturales que ellas implican para los grupos involucrados y su entorno.

Esta investigación tiene por objetivo indagar las tecnologías socioculturales desplegadas por migrantes por estilo de vida en la ciudad de Pucón e indagar en qué medida ellas contribuyen a un proceso de innovación sustentado en el intercambio cultural entre los migrantes y los habitantes de las comunidades receptoras desde la visión de los migrantes emprendedores. 


\section{La migración por insatisfacción como espacio de aprendizaje y experimentación}

La literatura de migración por estilo de vida ha relacionado los intentos por cambiar las condiciones de vida mediante la migración con un conjunto de variables que operan a nivel subjetivo y que emergen a partir de la situación de insatisfacción en que las personas se encuentran en el lugar de destino del movimiento, situación que se ha relacionado con las tensiones y contradicciones propias que se experimentan en las grandes ciudades contemporáneas (Benson, 2011; Zunino et al., 2013). Estos cambios están asociados a una transformación de las condiciones materiales de vida que muchas veces implica iniciar nuevas labores y emprendimientos, los cuales se relacionan en forma compleja y diversa con el entorno social y cultural local (Zunino et al., 2016). Para McIntyre (2009), esta forma de movilidad contemporánea implica una elección consciente dentro un universo de posibilidades y está condicionada por la imagen subjetiva que los migrantes forman de los lugares de acogida y su percepción sobre la potencialidad que ofrece el medio para realizar un vida más próspera, plena y satisfactoria.

Esta perspectiva ha sido desplegada para capturar el potencial de transformación y las tensiones que acarrean los migrantes en diversos países latinoamericanos incluyendo Chile (Zunino e Hidalgo, 2010; Zunino et al., 2013; Sánchez, 2014), Ecuador (Hayes, 2014), Costa Rica y Panamá (Janoshka, 2011, 2013). Los destinos turísticos de la NorPatagonia chilena como Puerto Varas y Pucón constituyen casos paradigmáticos para examinar los efectos de esta forma de movilidad. Los estudios empíricos muestran que la población migrante actúa como agente de transformación y contribuyen al desarrollo local (Espinoza et al., 2014), aunque el proceso no está exento de tensiones con la población local debido a la contraposición de significados e imaginarios (Vergara et al., 2013).

Desde la perspectiva de la migración por amenidad se ha examinado la migración de personas desde las grandes metrópolis a ciudades pequeñas o pueblos en el interior, sobre todo destinos de montaña, cuya calidad ambiental y cultural es percibida como superior a la de sus antiguos lugares de residencia (Otero, 2009). Este fenómeno de contra-urbanización (migración ciudad-campo) ha sido analizado recientemente en varias regiones montañosas del mundo, como Estados Unidos, Canadá, Costa Rica, Argentina, los Alpes Europeos, Noruega, Suecia, Filipinas, Australia y Nueva Zelandia (ver las contribuciones en Moss, 2006). Los estudios efectuados en Costa Rica (Chaverri, 2006), Chile (Borsdorf e Hidalgo, 2009), (Marchant y Rojas, 2015) y Argentina (Otero et al., 2006, 2008, González et al., 2009, Otero y González, 2014) muestran importantes flujos poblacionales hacia lugares dotados de atributos naturales y culturales singulares, situación que sugiere la importancia que están adquiriendo la migraciones por amenidad en América Latina.

Si bien las dos formas de movilidad examinadas difieren en cuanto a si enfatizan las condiciones en el lugar de origen que motivan la búsqueda de nuevas perspectivas individuales o grupales (migración de estilo de vida) o en las condiciones en el medio receptor que atraen a migrantes (migración por amenidad), ambas se focalizan en un grupo poblacional que cuenta con los recursos económicos para la búsqueda intencionada de un lugar para realizar una forma de vida distinta respecto a la que dejan atrás. A pesar que hay mucho para develar todavía de las posibles rupturas de los migrantes en sus entornos de vida anteriores, Matossian et al. (2014) conceptua- 
lizan las migraciones por amenidad y por estilos de vida de manera conjunta, como «migración por insatisfacción».

En este tipo de migraciones en busca de una nueva vida por insatisfacción con la anterior o con el ambiente donde ella se desarrollaba, los individuos buscan aprender a través de las vivencias, en el curso de la vida, lo que Jarvis (2009) denomina lifelong learning. Todo el aprendizaje, para el autor, es social y toda la experiencia adquirida a través de movilidad (turismo, migración, movilidad espacial cotidiana, movilidad social, etc.) es altamente valorada por los seres humanos, dado que impacta en su desarrollo como personas y sujetos reflexivos (Córdoba Cejudo, 2007; Jarvis, 2012). Muchas veces, una experiencia de migración es vista por los migrantes de amenidad y por estilos de vida como un ejercicio de aprendizaje a través de la inserción en un territorio nuevo y distinto que les plantea una serie de desafíos de cambio y adaptación (Janoschka, 2011; Sardinha, 2013; Matossian et al., 2014: 242). Este animarse voluntariamente u obligadamente a adaptarse o re inventarse constituye el punto de partida de una serie de intervenciones en la realidad que son el eje de análisis de este estudio.

En principio, partimos por entender la innovación como todo pensamiento creativo que llevado a la acción desafía las creencias y prácticas establecidas en un determinado contexto, aportando nuevos valores. Nos interesa centrarnos en las dimensiones constitutivas de esa emergencia e implementación de visiones y acciones de los migrantes por estilo de vida, que mediante sus estrategias de vinculación social con las comunidades de llegada irrumpen con formas de ser, pensar y hacer que cubren necesidades insatisfechas en las mismas.

\section{Las tecnologías como dimensiones de análisis de la innovación sociocultural}

Todas nuestras intervenciones en la sociedad dan cuenta de nuestra visión del mundo y de cómo entendemos al otro. Los migrantes por estilos de vida, son sujetos que en muchas ocasiones tienen trayectorias de vida que han recorrido y compartido muchos escenarios en la resolución de sus búsquedas personales. Esto hace que sus tiempos de vida parezcan más densos en términos de experiencias vividas y sus visiones y costumbres den cuenta de una acumulación de trayectorias que hagan de ellos sujetos que parecen estar o ser de otro tiempo. Esto significa que es común que no sean parte de las corrientes principales de pensamiento y que sus valores, hábitos y costumbres en muchas ocasiones se perciban como diferentes de los de los habitantes de las localidades que los reciben. Así estas divergencias en sus estrategias de supervivencia e inserción en la sociedad local tienen un correlato en la trama simbólico-significativa en la cual se insertan. (Margulis et al., 2014). Por lo que pueden ser consideradas disruptivas en términos culturales. El estudio de las innovaciones socioculturales, por lo tanto, debe abordarse desde la pregunta por la producción de sentido y desde una concepción multidimensional de la experiencia humana. (Najmanovich, 2001: 4)

En toda innovación es preciso considerar de manera articulada el contexto de emergencia del sujeto que lidera el proceso de innovación (el emprendedor social) y el contexto de emergencia de la innovación. Tanto las personas como el contexto se circunscriben a un tiempo y un espa- 
cio que se vincula (Najmanovich, 2001: 7) ${ }^{5}$. De manera que la interacción, el contacto con otros diversos son una de las características de los entornos asociados a los procesos creativos y de innovación.

Los procesos de innovación social pueden ser vistos como el resultado de un proceso de aprendizaje colectivo que proporciona soluciones novedosas para satisfacer demandas y necesidades sociales. Así la innovación social es “una práctica, una actividad caracterizada por desarrollarse en red, de manera participativa, que tiene como resultado el empoderamiento de individuos y grupos, el aprendizaje y bienestar. Tiene su origen en las interacciones entre los individuos, las organizaciones y sus respectivos entornos operativos, interacciones que son posibles mediante el uso de la tecnología, que fomenta la constitución de estas redes, generando una mayor variedad de agentes que producen cambios profundos en las rutinas básicas, los recursos, los flujos de autoridad o las creencias/relaciones de cualquier sistema social contribuyendo a la aparición de nuevos modelos de desarrollo sostenibles" (Maracaida y Zanfardini, 2016). A diferencia de la innovación convencional, que se concentra en objetivos económicos orientados al aumento del lucro, la innovación social se preocupa por alcanzar metas sociales, culturales y políticas. La innovación social no es producida exclusivamente por expertos o científicos, sino que incluye conocimientos prácticos derivados de la experiencia.

Es posible definir Tecnología Social (Thomas, s/f) como:

"una forma de diseñar, desarrollar, implementar y gestionar tecnología orientada a resolver problemas sociales y ambientales, generando dinámicas sociales y económicas de inclusión social y de desarrollo sustentable. La Tecnología Social alcanza un amplio abanico de producciones de tecnologías de producto, proceso y organización: alimentos, vivienda, energía, agua potable, transporte, comunicaciones, entre otras. Los actores fundamentales de los procesos de desarrollo de Tecnologías Sociales en América Latina son: movimientos sociales, cooperativas populares, ONGs, unidades públicas de I+D, divisiones gubernamentales y organismos descentralizados, empresas públicas y, en menor medida, empresas privadas".

Considerando la importancia de las redes en cuanto a forma de vehiculizar aprendizajes y experiencias de los movimientos sociales se privilegia los emprendimientos contextuales, interactivos y éticos. Es importante destacar que en el caso de los emprendedores sociales, se valora la visión De Souza et al. (2007:10) que plantea que el foco de la innovación es principalmente cambiar las personas que cambian las cosas. Según De Souza et al. (2005) a partir de la metáfora de la 'trama' de vida, surge otro 'régimen de verdades' sobre qué es y cómo funciona la realidad. Bajo la prevalencia de la revolución cultural, dicho régimen de verdades se materializa en una visión contextual de mundo donde el contexto es la referencia, la interacción la estrategia y la ética el garante de la sostenibilidad de todas las formas y modos de vida en el planeta.

La sociología observa la tensión existente entre las estructuras (heredadas y coercitivas) y la agencia del sujeto. Bourdieu es quien logra bajo el concepto de habitus hacer una síntesis integrada de ambas perspectivas (estructuralismo y subjetivismo). De acuerdo a Bourdieu, el habitus es lo social incorporado desde una posición social (estructura estructurada) y principio generador de prácticas (estructura estructurante). Si bien el habitus es un "sistema de disposiciones durables" se reconoce en él la posibilidad de cambio, a partir de circunstancias, inquietudes personales y el deseo de cambiar. No obstante, la perspectiva de análisis de Bourdieu no logra avanzar en la dinámica vincular que interesa a los fines de la innovación cultural. 
Según De Souza et al. (2006) el modo contextual corresponde la filosofía de innovación de cambiar las personas que cambian las cosas. Diferente de las cosas que pueden ser cambiadas por decreto, el arte de cambiar las personas exige el manejo de ciertas tecnologías (intelectual, social, cultural, política y de la sabiduría) que facilitan el proceso del cambio conceptual-cambio del régimen de verdades sobre qué es y cómo funciona la realidad:

- La tecnología intelectual es la tecnología de la interpretación, que maneja ideas, conceptos, teorías, paradigmas, metáforas y analogías para comprender la realidad que queremos transformar, como la realidad personal, profesional e institucional.

- La tecnología social es la tecnología de la interacción humana, que incluye métodos participativos, técnicas de negociación, enfoques para el manejo de conflictos y marcos para el aprendizaje social.

- La tecnología cultural es la tecnología de la construcción de significados que dan sentido a la existencia, que incluye métodos de convivencia intercultural de grupos y comunitarias, de deconstrucción, descolonización y reconstrucción de modos de interpretación y modos de intervención.

- La tecnología política es la tecnología del poder, que negocia criterios y valores para decidir sobre la inclusión y la exclusión, solidaridad y egoísmo, sensibilidad e indiferencia, regímenes democráticos y autoritarios, individualismo y colectivismo.

- La tecnología de la sabiduría es la tecnología de la vida, que incluye un comportamiento consciente y comprometido con ciertas prácticas que cultivan las condiciones, relaciones y significados que generan, sostienen y dan sentido a la existencia de todas las formas y modos de vida en el planeta.

A los fines de este estudio se definen las tecnologías socioculturales como aquellas tecnologías de la interacción humana, que incluyen métodos participativos, técnicas de negociación, enfoques para el manejo de conflictos y marcos para el aprendizaje social que construyen significados a la existencia a partir del mundo de la vida, para generar dinámicas sociales y económicas que promuevan la inclusión social y el desarrollo sustentable.

\section{Estrategia metodológica y caso de estudio}

Pucón es uno de los principales centros turísticos del sur de Chile. Es una comuna de la Provincia de Cautín perteneciente a la Región de la Araucanía, situada en el sur de Chile. Ubicada a $110 \mathrm{~km}$ de al sudeste de Temuco y a $780 \mathrm{~km}$ al sur de Santiago (ver Figura № 1).

La ciudad de Pucón ubicada sobre la costa oriental del lago Villarrica, se destaca por ser el balneario lacustre más importante de Chile, con gran actividad de temporada en la época estival. En su entorno tiene una variedad de atractivos turísticos que complementan sus playas sobre el lago, como centros termales, pistas para diversidad de actividades de nieve y senderos en parques nacionales.

Según el Censo del año 2002 Pucón tiene 21.107 habitantes, siendo una de las pocas comunas a nivel país con un crecimiento demográfico que supera la media nacional en los últimos 10 años (Municipalidad de Pucón, 2010). La variación intercensal en el período 1992-2002 alcanzó el 47\%, con un fuerte incremento poblacional en el área urbana: 72,4\% (Zunino y Hidalgo, 2010: 7). El ca- 
Figura No 1

Localización del área de estudio

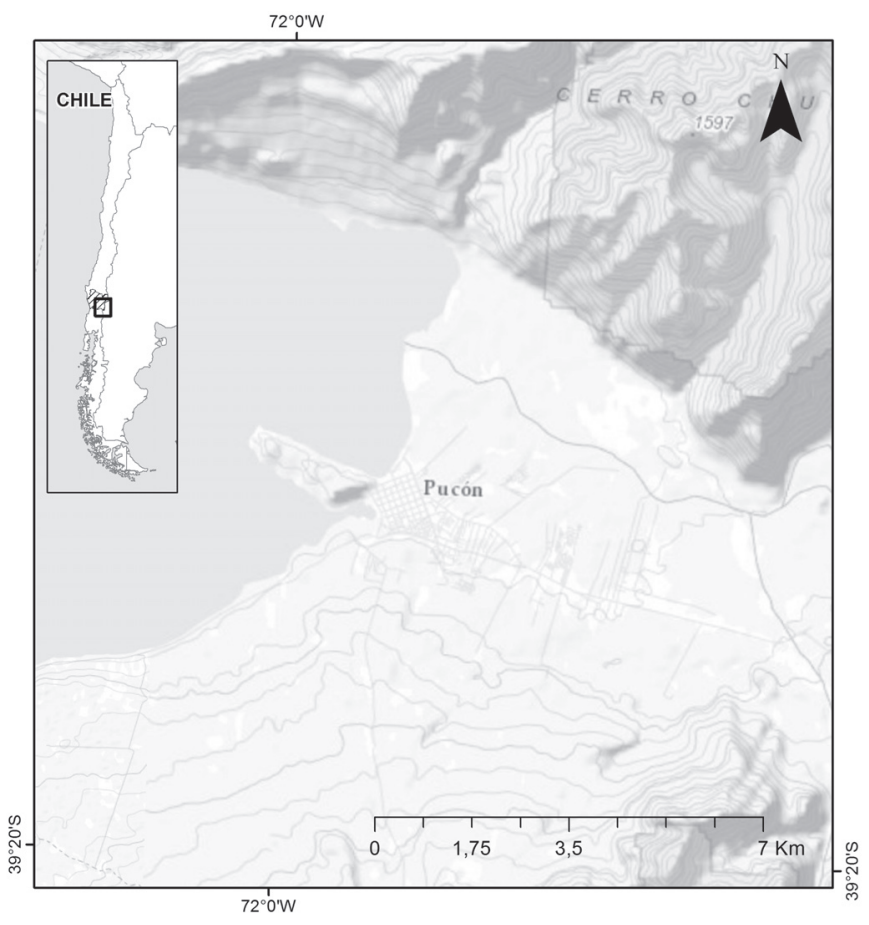

Fuente: Elaboración propia.

rácter social y cultural de la ciudad se ha transformado radicalmente por la llegada de migrantes por estilo de vida, los cuáles engloban variados personajes incluyendo intelectuales buscando fuentes de inspiración, bohemios en busca de diversión y vida agitada, extranjeros reinventado sus vidas en los confines del mundo, neo-hippies y místicos en búsqueda del yo personal e individuos de clase media buscando reinventar su relación consigo mismo y el entorno (Hidalgo et al., 2014; Zunino et al., 2014).

Zunino et al. (2011: 17-18) enfatizan el carácter cosmopolita y ecléctico de la ciudad, que está dado por la presencia de migrantes por estilo de vida de diversas nacionalidades y por la presencia de diversos emprendimientos alternativos, incluyendo pintorescos cafés, restaurants, hostales temáticos, templos budistas y centros de sanación. Dichos autores también destacan lo febril de la actividad turística en la época estival y el despliegue de estrategias de distintos organismos público-privados interesados en mantener el atractivo turístico del lugar, donde se da origen a una "ciudad fachada" especialmente fabricada para los turistas. La ciudad, así, representa un laboratorio natural para estudiar procesos de innovación y emprendimiento.

En esta investigación estamos interesados en estudiar las tecnologías socioculturales para una cultura de innovación aportada por los migrantes de amenidad y por estilos de vida que se han asentado en la ciudad de Pucón en los últimos 10 años. La unidad de análisis son las tecnolo- 
gías socioculturales presentes en los procesos de innovación. La muestra no probabilística se seleccionó realizando un barrido inicial de los principales emprendimientos que cumplieran con el criterio de representar innovaciones en el contexto social y cultural de la localidad, incluyendo en la muestra proyectos constructivos (construcción en barro y construcción solidaria), proyectos de medios de comunicación alternativos a las grandes cadenas, proyectos artístico-culturales, proyectos educativos (escuelas experimentales, escuelas Waldorf), proyectos productivos comunitarios y proyectos ambientales. Las unidades de relevamiento se estructuran en base a 12 migrantes de amenidad y por estilo de vida que hayan participado de procesos de innovación sociocultural en la localidad en estudio o en su radio de influencia (ver Cuadro $N^{\circ} 1$ ).

Cuadro No 1

Caracterización de unidades de relevamiento

\begin{tabular}{|c|c|c|c|c|c|}
\hline $\begin{array}{c}\text { Unidad de } \\
\text { Relevamiento } \\
\text { (UR) }\end{array}$ & Edad & $\begin{array}{l}\text { Nacionalidad } \\
\text { y Procedencia }\end{array}$ & $\begin{array}{l}\text { Residencias } \\
\text { Anteriores }\end{array}$ & $\begin{array}{c}\text { Máximo Nivel } \\
\text { de Instrucción } \\
\text { Alcanzado y } \\
\text { Título }\end{array}$ & Empleo Actual \\
\hline 1 & 39 & $\begin{array}{l}\text { Chileno } \\
\text { (Temuco) }\end{array}$ & $\begin{array}{c}\text { Tirúa } \\
\text { Comunidades } \\
\text { de México y } \\
\text { Brasil }\end{array}$ & $\begin{array}{l}\text { U.C.: Técnico } \\
\text { Electrónico }\end{array}$ & $\begin{array}{c}\text { Maestro Escuela } \\
\text { Waldorf }\end{array}$ \\
\hline 2 & 37 & $\begin{array}{l}\text { Alemán } \\
\text { (Munich) }\end{array}$ & $\begin{array}{c}\text { Arica } \\
\text { Santiago }\end{array}$ & $\begin{array}{c}\text { U.C. } \\
\text { Administrador de } \\
\text { Empresas }\end{array}$ & $\begin{array}{c}\text { Coordinador de } \\
\text { un programa de } \\
\text { Educación Ambiental } \\
\text { en una ONG }\end{array}$ \\
\hline 3 & 45 & $\begin{array}{c}\text { Chileno } \\
\text { (Valparaíso) }\end{array}$ & $\begin{array}{l}\text { Santiago } \\
\text { París }\end{array}$ & $\begin{array}{l}\text { U.C. Ingeniero } \\
\text { Agrónomo }\end{array}$ & $\begin{array}{c}\text { Socio Propietario de } \\
\text { Eco Hostel }\end{array}$ \\
\hline 41 & 40 & $\begin{array}{l}\text { Chileno } \\
\text { (Santiago) }\end{array}$ & $\begin{array}{c}\text { Talca } \\
\text { Coyhaique }\end{array}$ & $\begin{array}{l}\text { U.C. Trabajador } \\
\text { Social }\end{array}$ & $\begin{array}{c}\text { Emprendedor de } \\
\text { Empresa B }\end{array}$ \\
\hline 5 & 35 & $\begin{array}{l}\text { Chileno } \\
\text { (Santiago) }\end{array}$ & Bolivia & $\begin{array}{l}\text { U.C. Ingeniero } \\
\text { Comercial }\end{array}$ & Consultor en Turismo \\
\hline 6 & 43 & $\begin{array}{l}\text { Chilena } \\
\text { (Curicó) }\end{array}$ & $\begin{array}{l}\text { Curicó, } \\
\text { Santiago, } \\
\text { Escocia }\end{array}$ & U.C.: Veterinaria & $\begin{array}{c}\text { Maestra Escuela } \\
\text { Waldorf }\end{array}$ \\
\hline 7 & 45 & $\begin{array}{l}\text { Británico } \\
\text { (Inglaterra) }\end{array}$ & Santiago & $\begin{array}{c}\text { U.C. } \\
\text { Ingeniero } \\
\text { Agrónomo }\end{array}$ & $\begin{array}{l}\text { Propietario de } \\
\text { restaurante }\end{array}$ \\
\hline 8 & 37 & $\begin{array}{c}\text { Chileno } \\
\text { (Santiago) }\end{array}$ & $\begin{array}{c}\text { Melbourne } \\
\text { San Francisco } \\
\text { Mallorca }\end{array}$ & $\begin{array}{c}\text { U.C. } \\
\text { Licenciado en } \\
\text { Turismo }\end{array}$ & Tour Operador \\
\hline 9 & 35 & Alemana & $\begin{array}{c}\text { Chile } \\
\text { Alemania }\end{array}$ & Universitario & $\begin{array}{c}\text { Propietaria de } \\
\text { un hostel y tour } \\
\text { operadora }\end{array}$ \\
\hline 10 & 62 & $\begin{array}{l}\text { Británico } \\
\text { (Escocia) }\end{array}$ & Antártida & U.C.: Derecho & Reikista \\
\hline
\end{tabular}


Continuación Cuadro No 1

\begin{tabular}{|c|c|c|c|c|c|}
\hline $\begin{array}{c}\text { Unidad de } \\
\text { Relevamiento } \\
\text { (UR) }\end{array}$ & Edad & $\begin{array}{c}\text { Nacionalidad } \\
\text { y Procedencia }\end{array}$ & $\begin{array}{c}\text { Residencias } \\
\text { Anteriores }\end{array}$ & $\begin{array}{c}\text { Máximo Nivel } \\
\text { de Instrucción } \\
\text { Alcanzado y } \\
\text { Título }\end{array}$ & Empleo Actual \\
\hline 11 & 72 & $\begin{array}{c}\text { Colombiano } \\
\text { (Cali) }\end{array}$ & Estados Unidos & U.I. & $\begin{array}{c}\text { Propietario de } \\
\text { Agencia de Viajes }\end{array}$ \\
\hline 12 & 50 & $\begin{array}{c}\text { Chileno } \\
\text { (Santiago) }\end{array}$ & $\begin{array}{c}\text { Valdivia } \\
\text { Villarrica }\end{array}$ & $\begin{array}{c}\text { Administrador de } \\
\text { Empresas }\end{array}$ & $\begin{array}{c}\text { Director de una } \\
\text { fundación que } \\
\text { concientiza sobre el } \\
\text { uso de energías no } \\
\text { convencionales }\end{array}$ \\
\hline
\end{tabular}

Nota: (U.C.) Universitario Completo / (U.I.) Universitario Incompleto.

Fuente: Elaboración propia.

Se realizaron en total doce (12) entrevistas en profundidad durante el mes de Junio de 2015 en la ciudad de Pucón con el migrante-emprendedor que lideraba la iniciativa de innovación seleccionada. Las entrevistas incluyeron diecisiete (17) preguntas, de las cuales nueve (9) buscaban obtener un perfil socio demográfico de los entrevistados y capturar las experiencia de la población migrante-emprendedora, incluyendo dimensiones tales como edad, las experiencia de vida temprana del sujeto (niñez y juventud); los intereses, preocupaciones y experiencias en su etapa adulta, su participación en grupos sociales, los lugares donde había residido, las razones de porque había elegido a Pucón como lugar de residencia, sus estudios y experiencias laborales. Y otras ocho (8) preguntas orientadas a profundizar en su vínculo con su emprendimiento, tales como el origen y características del mismo, sus objetivos sociales, sus criterios para incluir otras personas en el mismo, el aporte de las culturas tradicionales, su participación en redes de cooperación y como el sujeto visualiza su contribución a la sociedad. Si bien estas preguntas directrices enmarcaron la entrevista, el diálogo fue de carácter abierto y permitiéndole al sujeto explayarse en aquellos puntos importantes para él o ella. Las entrevistas tuvieron una duración promedio de 2 horas cada una.

Las entrevistas se analizaron, ordenando las respuestas en una matriz de datos según las distintas dimensiones de análisis, y a través de la inducción analítica se buscó resaltar características comunes en las perspectivas de los entrevistados y compararlas en la búsqueda de emergentes y posibles patrones comunes. La investigación se enmarcó en el paradigma cualitativo ya que buscaba estudiar una realidad social compleja, donde el interés clave fue comprender las tecnologías socio culturales presentes en los procesos de innovación desde la perspectiva de los migrantes emprendedores. Su carácter fue exploratorio, dado que el objetivo era dar luz a cuáles a dimensiones críticas iniciales en relación al proceso de producción de las innovaciones socioculturales. 


\section{La perspectiva de los Migrantes por Estilo de Vida de Pucón en relación a sus innovaciones socioculturales}

Se presenta el análisis de resultados a partir del Cuadro $N^{\circ} 2$, que da cuenta de las opiniones relevantes de los migrantes emprendedores en relación al objeto de este estudio. Las mismas fueron analizadas a la luz de las distintas dimensiones de las tecnologías socioculturales presentadas en el apartado teórico. A partir de ello, ha sido posible identificar una serie de categorías de análisis emergentes para cada una de las dimensiones teóricas. A continuación se desarrollan algunas ideas que permitirán comprender mejor la vinculación entre los marcos analíticos y las categorías de análisis presentadas.

- Los migrantes de amenidad y por estilos de vida entrevistados han referido a la importancia del contexto, en este caso la ciudad de Pucón como un lugar donde es posible recrear los intereses y valores personales a través de la "creación de atmósferas", entendiendo por tales la existencia y el intercambio con pares o personas con afinidad de valores e intereses.

- En sus estrategias de acciones socioculturales los migrantes por estilos de vida valoran la interacción y el intercambio más allá de las reglas del mercado, les interesa generar intercambios de carácter simétrico, donde ambas partes ganen, por ejemplo a través de trueques de productos orgánicos, generando redes de comercialización para productores provenientes de lugares con dificultades para llegar al mercado.

- La gente comparte visiones y las redes ayudan a sostenerlas, en este sentido el poner a la gente a conversar, generar interacciones para la creación de capital social es una tecnología sociocultural crítica, dado que constituyen andamiajes para proyectos compartidos o para la defensa de valores comunes. Así en Pucón, el Movimiento Pucón en Transición ha sido una manifestación colectiva de muchos migrantes de amenidad y por estilos de vida que junto a lugareños emprendieron una cruzada por cuidar y mejorar sus prácticas individuales y colectivas en pos de una mejor calidad de vida en la localidad.

- La necesidad de una visión de la complejidad en el aprendizaje para el cambio es un elemento considerado efectivo en la generación de capacidades sociales.

- La capacidad de escucha es una tecnología social básica para la interacción y por supuesto para el inicio del proceso de innovación.

- Los migrantes por estilo de vida presentan como tecnología social la capacidad para detectar "anomalías" a las que se consagran y se constituyen en el motor de sus proyectos.

- Las tecnologías socioculturales para la innovación son producto de la emergencia de lo nuevo, como acumulación histórica de horizontes y prácticas.

- Los emprendedores sociales son personas que generan innovaciones porque son capaces de pasar de las ideas a la acción, y tratan de ser consecuentes con sus búsquedas. En distintas dimensiones, especialmente en el área social los emprendedores tienen un foco, es decir, logran focalizarse. 
- Las tecnologías socioculturales incluyen métodos participativos de interacción humana que impactan en la mejora de problemas de conflictividad social.

- En su hacer los innovadores socioculturales generan procesos de resignificación de las tecnologías de la sabiduría, intelectuales y sociales en su búsqueda de lograr sintonía con las realidades, necesidades y aspiraciones con los actores de la comunidad.

- Un tema valorado como central en las tecnologías de las innovaciones socioculturales es trabajar para la construcción duradera de capital social, y en muchos casos esto tiene que ver con la interacción y el tiempo de permanencia con el otro.

- En relación a las tecnologías de la sabiduría, un cambio de paradigma en relación a la cosmovisión de la vida actual plantea una armonía entre el ser y el hacer.

- Otra cuestión que se considera importante en el cruce entre innovadores socioculturales y migrantes de amenidad y por estilos de vida, es que ellos buscan que sus emprendimientos sean coherentes con el estilo de vida que ellos están buscando y que ello les devuelva armonía y felicidad para sus vidas.

- Los aprendizajes para el cambio en los emprendedores sociales se plantean como un idea y vuelta entre el hacer y la necesidad de nuevos saberes necesarios para avanzar en problemáticas focalizadas que pretenden resolver.

- Una de las tecnologías sociales que los innovadores sociales deben tener es la posibilidad de ser capaces de generar visibilidad en su interacción con su medio social, ya sea real o virtual por sus ideas de proyectos o de sus innovaciones.

- Las tecnologías socioculturales de los migrantes de amenidad también afectan la oferta de productos turísticos en Pucón como destino turístico, ya que se generan nuevas experiencias turísticas en su área de influencia, a partir de la generación de situaciones que buscan el aprendizaje o bien la transformación personal y en otros casos colaboran con la conservación del patrimonio de las áreas.

- Si se consideran las tecnologías sociales como formas de diseñar, desarrollar, implementar y gestionar tecnología orientada a resolver problemas sociales y ambientales, vemos que los emprendedores socioculturales trabajan para generar ahorros sociales, como en relación a la contaminación ambiental y mejorar las condiciones de vida de las personas, de la flora y de la fauna. 


\section{Cuadro $\mathrm{N}^{\circ} 2$}

La perspectivas de los migrantes por Estilo de vida de Pucón en relación a sus innovaciones socioculturales

\begin{tabular}{|c|c|c|}
\hline Tecnología & $\begin{array}{l}\text { Categoría de } \\
\text { Análisis }\end{array}$ & $\begin{array}{c}\text { Perspectiva de los Migrantes por Estilo de vida de Pucón } \\
\text { de sus innovaciones socioculturales }\end{array}$ \\
\hline \multirow[t]{4}{*}{$\begin{array}{l}\text { De la } \\
\text { interpretación } \\
\text { (Intelectual) }\end{array}$} & $\begin{array}{l}\text { Generación de } \\
\text { pensamiento } \\
\text { ecologizante }\end{array}$ & $\begin{array}{l}\text { Todas estas metodologías son como cosas que hemos } \\
\text { ido pasando en limpio... una metodología propia y que } \\
\text { la hemos ido probando,...y poco a poco se fue transfor- } \\
\text { mando a ser una facilitación, ...., porque podís tener un } \\
\text { producto turístico en un mapa súper articulado, pero si } \\
\text { no hay procesos que hagan que funcione y no hay una } \\
\text { vitalidad, y tampoco tienes un propósito común de este } \\
\text { organismo ....Eso es lo que hemos ido aprendiendo, } \\
\text { cómo sacar paralelos de los organismos vivos para que } \\
\text { las organizaciones sean vivas, o sea organizaciones que } \\
\text { aprenden, y no sean cadáveres de papel"..(UR5) }\end{array}$ \\
\hline & $\begin{array}{l}\text { Generación de } \\
\text { pensamiento } \\
\text { complejo }\end{array}$ & $\begin{array}{l}\text { "...La tercera yo diría que es la capacidad de evaluar, } \\
\text { permanentemente de evaluar y de, no necesariamente } \\
\text { ponerle métrica a la evaluación en el inicio, pero de eva- } \\
\text { luar sistemáticamente. Entonces aquí el emprendedor se } \\
\text { puede cambiar de caballo, digo yo, igual que el sentido } \\
\text { común yo voy en este caballo y me cambio de caballo } \\
\text { porque digo mira en realidad la cosa no va por acá." (UR4) }\end{array}$ \\
\hline & $\begin{array}{l}\text { Capacidad para } \\
\text { detectar anomalías }\end{array}$ & $\begin{array}{l}\text { "...siempre tenemos que nutrir con un tema de emprendi- } \\
\text { miento, un tema de diseño, un tema de trabajo social o co- } \\
\text { laborativo y el desarrollo personal a través del aprendizaje, } \\
\text { entonces así sentimos que vamos llegando a las diferentes } \\
\text { necesidades de un grupo. Y después cada persona va en- } \\
\text { tendiendo cuál es su rol y dónde tiene un posible liderazgo } \\
\text { dentro de estos ámbitos necesarios de desarrollo." (UR5). }\end{array}$ \\
\hline & $\begin{array}{l}\text { Capacidad de } \\
\text { realización }\end{array}$ & $\begin{array}{l}\text { "...Me tinca como decimos los chilenos, me late que... en- } \\
\text { tonces, lo que un experto está obligado a fundamentar el } \\
\text { emprendedor no está obligado a fundamentar. Entonces } \\
\text { dice me late que esta cosa es por acá.... entonces por } \\
\text { qué es tan relevante este punto, porque... yo diría que en } \\
\text { la literatura está bien ausente, los emprendedores son } \\
\text { emprendedores porque están consagrados a anomalías, } \\
\text { no a problemas. Los expertos están consagrados a pro- } \\
\text { blemas" (UR4) } \\
\text { "... Yo creo que una de las contribuciones que he hecho, } \\
\text { pero que es más pa mí, pero que igual ayuda, es hacer } \\
\text { lo que pensaba. O sea terminé construyéndome mi casa } \\
\text { como yo quería, construí... logramos hacer un barrio en- } \\
\text { tre amigos, el colegio está funcionando. Entonces el lle- } \\
\text { var a cabo proyectos que en un momento eran sueños } \\
\text {....y después de estos años verlos concretizados creo que } \\
\text { es un aporte" (UR5). }\end{array}$ \\
\hline
\end{tabular}


Continuación Cuadro No 2

\begin{tabular}{|c|c|c|}
\hline Tecnología & $\begin{array}{l}\text { Categoría de } \\
\text { Análisis }\end{array}$ & $\begin{array}{l}\text { Perspectiva de los Migrantes por Estilo de vida de Pucón } \\
\text { de sus innovaciones socioculturales }\end{array}$ \\
\hline \multirow[t]{5}{*}{$\begin{array}{l}\text { De la } \\
\text { Interacción } \\
\text { humana (Social) }\end{array}$} & $\begin{array}{l}\text { Creación de } \\
\text { Atmosferas }\end{array}$ & $\begin{array}{l}\text { “...yo creo que una de las grandes razones de que conti- } \\
\text { núe apoyando... bueno, porque a mí me inspira el proyec- } \\
\text { to en sí, pero porque siento que hay un grupo de trabajo } \\
\text { que me nutre mucho a mí en que está ... y hay otros más } \\
\text { que están ahí, yo siento que tiene una fuerza cohesiva } \\
\text { muy fuerte." (UR5). }\end{array}$ \\
\hline & $\begin{array}{l}\text { Capacidad de } \\
\text { escucha }\end{array}$ & $\begin{array}{l}\text { Escuchar desde la dimensión de suspenderme, suspender } \\
\text { mis preocupaciones, mis ofertas, mis interpretaciones... el } \\
\text { escuchar preocupaciones ¿en qué sentido? En el sentido } \\
\text { de pre-ocupación, es decir, todo ser humano está pre-ocu- } \\
\text { pado, todo ser humano está por lo tanto en un intersticio } \\
\text { en que va para el futuro pero está hoy día y algo quiere } \\
\text { hacer y uno dice bueno entonces yo en el presente puedo } \\
\text { hacerme cargo de esas preocupaciones, de esos intere- } \\
\text { ses que mueven a las personas. Yo te diría que eso ha sido } \\
\text { fundamental para mí... el tercer aspecto del escuchar o di- } \\
\text { mensión del escuchar: se escuchan las tendencias." (UR4). }\end{array}$ \\
\hline & $\begin{array}{l}\text { Mejora de las } \\
\text { condiciones } \\
\text { sociales } \\
\text { (conflictos) y } \\
\text { ambientales }\end{array}$ & $\begin{array}{l}\text { "Pero nosotros en un verano, podemos reciclar treinta } \\
\text { toneladas de residuos orgánicos y cuando empieza a di- } \\
\text { mensionar que si no hiciéramos eso mandaríamos cin- } \\
\text { cuenta toneladas de residuos a la basura. Entonces te } \\
\text { das cuenta iguau! Y además todo lo reciclable se dona, } \\
\text { se regala para fortalecer las familias que se dedican a } \\
\text { eso en Curarrehue,..." “...H. fue el que durante el período } \\
\text { militar fue el que trajo dinero del mundo para el movi- } \\
\text { miento ambiental; H. fue el que paró la caza de ballenas } \\
\text { en Chile; H. fue el que trajo la norma FCC (la más im- } \\
\text { portante norma de protección de bosques del mundo a } \\
\text { Chile) (UR2). }\end{array}$ \\
\hline & $\begin{array}{l}\text { Generar } \\
\text { intercambios } \\
\text { simétricos }\end{array}$ & $\begin{array}{l}\text { "...el año pasado se hizo un plan en escuelas rurales para } \\
\text { niños, ...los resultados fueron que la directora nos decía } \\
\text { que el bullying disminuyó muchísimo... se hicieron char- } \\
\text { las, talleres, juegos, pintemos un... hagamos un mural, } \\
\text { hicieron caminatas, cantaron..."(UR2). } \\
\text { "....En ese sentido del intercambio, ellos nos dan sabor, } \\
\text { nosotros les ayudamos a comprar. Pero participamos en } \\
\text { cosas que son súper simétricas, como que vamos a limpiar } \\
\text { la ciudad... y nosotros hablamos de educación ambiental y } \\
\text { pagamos nuestro stand igual que los otros y..." (UR2). }\end{array}$ \\
\hline & $\begin{array}{l}\text { Generación de } \\
\text { conexiones críticas }\end{array}$ & $\begin{array}{l}\text { “...uno aprende y uno va enseñando, no... entonces por } \\
\text { eso por ejemplo tenemos una página web que se llama } \\
\text { Pucón en Transición, es una página en Facebook, y que } \\
\text { partió el } 2012 \text { y que ahora tiene } 3.200 \text { personas y es } \\
\text { como el punto de muchas iniciativas..." (UR2) }\end{array}$ \\
\hline
\end{tabular}


Continuación Cuadro No 2

\begin{tabular}{|c|c|c|}
\hline Tecnología & $\begin{array}{l}\text { Categoría de } \\
\text { Análisis }\end{array}$ & $\begin{array}{c}\text { Perspectiva de los Migrantes por Estilo de vida de Pucón } \\
\text { de sus innovaciones socioculturales }\end{array}$ \\
\hline \multirow[t]{6}{*}{$\begin{array}{l}\text { De la } \\
\text { Interacción } \\
\text { humana (Social) }\end{array}$} & & $\begin{array}{l}\text { "...trabajamos todo el tema de generar redes y comuni- } \\
\text { dades, pero redes de trabajo colaborativas desde nuevos } \\
\text { paradigmas valóricos y comunicacionales y el poder que } \\
\text { tiene la auto reflexión en torno al trabajo grupal, y esa auto } \\
\text { reflexión hace que... la gente se va dando cuenta de que } \\
\text { estamos con paradigmas muy competitivos de no colabo- } \\
\text { ración y que al final va en desmedro de cada uno..."(UR5). }\end{array}$ \\
\hline & \multirow[t]{2}{*}{$\begin{array}{l}\text { Generación de } \\
\text { aprendizajes } \\
\text { experienciales }\end{array}$} & $\begin{array}{l}\text { “...Las redes permiten eso. Las redes permiten insertarse } \\
\text { en la tradición con un costo menor de transacción... que } \\
\text { sus nodos le generen conexiones virtuosas, positivas, } \\
\text { que le aporten valor a la propuesta de él. Entonces las } \\
\text { redes son muy, muy, muy fundamentales... aquí no hay } \\
\text { masa crítica, nosotros decimos no nos importa eso por- } \\
\text { que nosotros no andamos buscando masa crítica, anda- } \\
\text { mos buscando conexiones críticas..."(UR4). }\end{array}$ \\
\hline & & $\begin{array}{l}\text { "...el otro tema tiene que ver con el rol que jugamos ...Y } \\
\text { lo que yo diría que es más permanente en el tiempo es lo } \\
\text { que les mencionaba yo del rol que jugamos como espa- } \\
\text { cio comunitario..."(UR3). }\end{array}$ \\
\hline & $\begin{array}{l}\text { Mejora de } \\
\text { procesos } \\
\text { productivos para } \\
\text { Desarrollo local }\end{array}$ & $\begin{array}{l}\text { "...Tenemos carpas, comida vegetariana de primera cali- } \\
\text { dad, ....en los distintos cursos de liderazgo, y proyectos } \\
\text { de vida que se dictan en la Reserva, todos ellos de nivel } \\
\text { avanzado... la gente repite las experiencias y nos comen- } \\
\text { ta a su regreso cómo han desarrollado sus proyectos per- } \\
\text { sonales a partir de los aprendizajes iniciales" (UR2). }\end{array}$ \\
\hline & $\begin{array}{l}\text { Construcción de } \\
\text { capital social }\end{array}$ & $\begin{array}{l}\text { "...el turismo de conservación... fue un proyecto que nos } \\
\text { contrató la WWF.... vamos y nos quedamos con una co- } \\
\text { munidad de pescadores ...Y esos pescadores están inten- } \\
\text { tando trabajar en turismo, pero no saben muy bien cómo, } \\
\text { entonces nosotros hemos tenido que ir a entrenarlos to- } \\
\text { das estas veces, y ahora vamos... vamos con esta gente. } \\
\text { En esa área hay un parque privado de conservación ...que } \\
\text { es de un grupo de científicos que no querían turistas, } \\
\text { pero nosotros los convencimos de recibir turistas para } \\
\text { financiar sus acciones de conservación, y ahí lo que ellos } \\
\text { conservan es el bosque y el pudú..." (UR8) }\end{array}$ \\
\hline & $\begin{array}{l}\text { Generación de } \\
\text { aprendizajes } \\
\text { tutoriales }\end{array}$ & $\begin{array}{l}\text { “...es una consultora especializada en turismo sostenible } \\
\text { y..., tenemos un área de capacitaciones de todo el tema } \\
\text { de cómo se implementan las buenas prácticas, el funda- } \\
\text { mento de la sustentabilidad, ...después tenemos todo el } \\
\text { tema de diseño de experiencia con su metodología ex- } \\
\text { periencial,...terminamos elaborando una metodología de } \\
\text { cómo hacerlo y cómo desarrollarlo..."(UR5). }\end{array}$ \\
\hline
\end{tabular}


Continuación Cuadro No 2

\begin{tabular}{|c|c|c|}
\hline Tecnología & $\begin{array}{l}\text { Categoría de } \\
\text { Análisis }\end{array}$ & $\begin{array}{c}\text { Perspectiva de los Migrantes por Estilo de vida de Pucón } \\
\text { de sus innovaciones socioculturales }\end{array}$ \\
\hline $\begin{array}{l}\text { De la } \\
\text { Interacción } \\
\text { humana (Social) }\end{array}$ & $\begin{array}{l}\text { Generación de } \\
\text { ahorros sociales }\end{array}$ & $\begin{array}{l}\text { "...Pero este otro proyecto tiene que ver, como te digo, } \\
\text { generar un encadenamiento productivo para que el sec- } \\
\text { tor gastronómico, pueda generar una propuesta culinaria } \\
\text { con identidad..." (UR3) } \\
\text { "...Lo que nosotros sabemos hacer es que sabemos desa- } \\
\text { tar procesos en donde el capital social permite, primero, } \\
\text { la generación de confianza a partir de decirse las des- } \\
\text { confianzas..."(UR4) } \\
\text { "....aparte de lo que era el taller, que igual era bien ex- } \\
\text { periencial, también había mucho tiempo de compartir y } \\
\text { de conocerse y de estar... salir de tu casa, de tu oveja y } \\
\text { de tu rollo, qué sé yo, e ir a otro espacio como un viaje } \\
\text { ¿cachay? un viaje de aprendizaje o de estudio, qué sé yo, } \\
\text { y..." ..."(UR5) } \\
\text { "...De plantearte ese proyecto como tu camino de apren- } \\
\text { dizaje, entonces es un aprendizaje en acción, tú vas ha- } \\
\text { ciendo acciones, vas revisando lo que vas haciendo y eso } \\
\text { tiene una metodología de evaluación con un par, con un } \\
\text { mentor. Tenía todo su cuento y después de dos años tú } \\
\text { presentas en el fondo tu aprendizaje en torno a un pro- } \\
\text { yecto real. Y eso siempre me gustó mucho, porque en el } \\
\text { fondo es una aplicación directa de cómo ir aprendiendo" } \\
\text { (UR5). } \\
\text { "...La gente ahorra muchísimo, porque a esas personas tú } \\
\text { le entregas la vivienda con un calefón, pero no siempre } \\
\text { tiene el dinero para comprar el gas. En cambio hoy dia } \\
\text { tiene } 250 \text { litros de agua caliente gratuita todos los días } \\
\text { con el sistema que inventamos..." (UR4) }\end{array}$ \\
\hline $\begin{array}{l}\text { De la } \\
\text { construcción de } \\
\text { significados } \\
\text { (Cultural) }\end{array}$ & $\begin{array}{l}\text { La emergencia de } \\
\text { lo nuevo como } \\
\text { acumulación } \\
\text { histórica de } \\
\text { horizontes y } \\
\text { prácticas }\end{array}$ & $\begin{array}{l}\text { "....ipor qué es tan interesante? Porque fíjate que eso es } \\
\text { lo que no advierten las compañías cuando son tan gran- } \\
\text { des, es decir, la innovación la ven como algo muy téc- } \\
\text { nico, muy Stanford, muy (...) y no logran darse cuenta } \\
\text { que... nosotros la definimos como la emergencia de lo } \\
\text { nuevo como la acumulación histórica de horizontes y } \\
\text { de prácticas. Y entonces nosotros decimos bueno pero } \\
\text { si la innovación está aquí mismo, entonces lo que hay } \\
\text { que hacer tener un buen observador que nos permita ver } \\
\text { cómo se mueven las personas, qué prácticas tienen, qué } \\
\text { prácticas asociativas tienen, qué miedos tienen. Enton- } \\
\text { ces en ese sentido le ayudamos a ellos a re configurar } \\
\text { ese espacio". (UR4). }\end{array}$ \\
\hline
\end{tabular}


Continuación Cuadro $N^{\circ} 2$

\begin{tabular}{|c|c|c|}
\hline Tecnología & $\begin{array}{l}\text { Categoría de } \\
\text { Análisis }\end{array}$ & $\begin{array}{c}\text { Perspectiva de los Migrantes por Estilo de vida de Pucón } \\
\text { de sus innovaciones socioculturales }\end{array}$ \\
\hline \multirow[t]{2}{*}{$\begin{array}{l}\text { De la } \\
\text { construcción de } \\
\text { significados } \\
\text { (Cultural) }\end{array}$} & $\begin{array}{l}\text { Rescate de la } \\
\text { cultura local y la } \\
\text { mezcla con otras } \\
\text { culturas }\end{array}$ & $\begin{array}{l}\text { Pero tal vez sí lo que hay ese eclecticismo, se derivan } \\
\text { ciertas formas de que esta organización busca tomar } \\
\text { como lo mejor de las tradiciones antiguas y lo mejor de } \\
\text { lo que te ofrece hoy día la sociedad. Es como esa conjun- } \\
\text { ción, y también como que ve la vida como un círculo que } \\
\text { tiene cuatro cuadrantes.... Entonces es una perspectiva } \\
\text { como integradora" (UR2). } \\
\text { "...Sí, hay una cocinera que es mapuche, y la verdad que } \\
\text { cocina espectacular, ... se va generando una suerte de } \\
\text { capacitación y se va capacitando gente que va entrando, } \\
\text { este mismo cabro que es comenzó como voluntario y es } \\
\text { gringo también aprende recetas autóctonas ¿te fijai? Ya } \\
\text { sabe ya cómo hacer los platos de acá, las mismas cocine- } \\
\text { ras les enseñan..." (UR3). }\end{array}$ \\
\hline & $\begin{array}{l}\text { Búsqueda de } \\
\text { trascendencia a } \\
\text { través de } \\
\text { Emprendimientos } \\
\text { Sociales }\end{array}$ & $\begin{array}{l}\text { "...Nosotros tenemos lo que llamamos las cultours, cul- } \\
\text { tours étnicos mapuche, que son tours que acercan la } \\
\text { gente a convivir con la comunidad mapuche..., yo creo } \\
\text { que es un tema de respeto. Tomé un curso de mapudun- } \\
\text { gun ... He tomado bastante tiempo en entender la cultu- } \\
\text { ra.... Y hay que entender la cultura y respetarla. Y noso- } \\
\text { tros hemos trabajado bastante con familias que hoy en } \\
\text { día trabajan también con otras empresas, ...fuimos den- } \\
\text { tro de los primeros que han estimulado y que también } \\
\text { damos opinión sin querer imponer" (UR9). } \\
\text { "...Entonces la gente queda feliz y todo, entonces nos va } \\
\text { alimentando ... además que es mucho... Lo más bonito } \\
\text { que tiene este trabajo es que al final nos alimenta mucho } \\
\text { a nosotros, porque vemos que la gente se va contenta, se } \\
\text { va re inspirada y eso te da una energía"(UR5). }\end{array}$ \\
\hline $\begin{array}{l}\text { Del Poder } \\
\text { (Política) }\end{array}$ & $\begin{array}{l}\text { Generar visibilidad } \\
\text { social }\end{array}$ & $\begin{array}{l}\text { "... A la hora de estar en las redes el tiempo es un fac- } \\
\text { tor fundamental, por ello debes ser capaz de transmitir } \\
\text { la idea de tu proyecto en el menor tiempo posible. ¿Sa- } \\
\text { bes qué es un elevator pitch? El elevator pitch toma su } \\
\text { nombre de una supuesta situación en la que, en lo que } \\
\text { dura un viaje en ascensor (menos de } 2 \text { minutos), debes } \\
\text { despertar el interés de tu interlocutor por tu proyecto; } \\
\text { ya sea un inversor, un cliente potencial o un posible cola- } \\
\text { borador... y por lo tanto la persistencia que la gente sepa } \\
\text { contar lo que hace de manera muy concreta, y si no es } \\
\text { concreta en el concepto tiene que saber emocionar ¿me } \\
\text { explico? (UR4). }\end{array}$ \\
\hline
\end{tabular}


Continuación Cuadro No 2

\begin{tabular}{|c|c|c|}
\hline Tecnología & $\begin{array}{l}\text { Categoría de } \\
\text { Análisis }\end{array}$ & $\begin{array}{c}\text { Perspectiva de los Migrantes por Estilo de vida de Pucón } \\
\text { de sus innovaciones socioculturales }\end{array}$ \\
\hline \multirow[t]{5}{*}{$\begin{array}{l}\text { De la vida } \\
\text { (Sabiduría) }\end{array}$} & \multirow[t]{2}{*}{$\begin{array}{l}\text { Cambio en el } \\
\text { régimen de } \\
\text { verdades }\end{array}$} & $\begin{array}{l}\text { "Yo llegué a una conclusión que lo que mejor puedo ha- } \\
\text { cer, es elaborar un esquema de valores humanos basado } \\
\text { en la tierra ....., es la conexión... eso de educar de manera } \\
\text { anónima es poco comprendido... El proyecto que a mí me } \\
\text { interesa es ir hilvanando un nuevo paradigma que no va } \\
\text { a venir de los gobiernos o las escuelas, de la educación } \\
\text { ni la religión ni nada, va a venir del interior de cada per- } \\
\text { sona..." (UR10). } \\
\text { “...O sea hemos notado cómo el cambio metodológico ha } \\
\text { cambiado los resultados en las personas de los talleres, } \\
\text { como lograr mucho más impacto, lograr más transforma- } \\
\text { ción en cuanto a la visión de mundo, y por lo tanto a una } \\
\text { acción más concreta que inspire y motive a hacer cosas... } \\
\text { y por eso le llamamos como esta metodología de apren- } \\
\text { dizaje transformativo" (UR5). }\end{array}$ \\
\hline & & $\begin{array}{l}\text { Nos enfocamos en el aprendizaje dinámico y vivencial, } \\
\text {....en ambientes donde la "experiencia", es como nuestro } \\
\text { gran recurso metodológico, y que juegue un rol altamen- } \\
\text { te significativo... se focaliza como una empresa de capa- } \\
\text { citación en coaching, en liderazgo, en trabajo en equipo } \\
\text { y que además trae líderes de distintas visiones de mundo } \\
\text { desde el sufismo, desde el budismo, desde el hinduismo, } \\
\text { es una perspectiva de abrir la cabeza a mucha gente" } \\
\text { (UR2). }\end{array}$ \\
\hline & \multirow[t]{3}{*}{$\begin{array}{l}\text { Una armonía entre } \\
\text { el ser y el hacer }\end{array}$} & $\begin{array}{l}\text { “... Entonces no es suficiente estar haciendo todo el tiem- } \\
\text { po porque nos quedamos atrapados en cosas que son } \\
\text { falsas... Pero tampoco sería suficiente simplemente ser } \\
\text { ¿no? ...Frank Sinatra lo tenía... tenía la idea bastante clara } \\
\text { en sus cantos, algunos cantos que en cada estrofa canta- } \\
\text { ba do be do be do...: hacer y ser, hacer y ser. Es simple- } \\
\text { mente asi" (UR10). }\end{array}$ \\
\hline & & $\begin{array}{l}\text { “...Y al generar ese quiebre, después de eso mostramos } \\
\text { una posible forma de desarrollo más armónica ¿ver- } \\
\text { dad?...todo esto tiene una cosa como bien humanista... } \\
\text { (UR5). }\end{array}$ \\
\hline & & $\begin{array}{l}\text { “...Ahora el interés que está naciendo en mí es el de la } \\
\text { consultoría en liderazgo orgánico. Como... cómo orga- } \\
\text { nizar, cómo poner el componente espiritual en organi- } \\
\text { zaciones para que la gente se pueda relacionar de una } \\
\text { manera que todos aprendan y que construyan ¿cachay?" } \\
\text { (UR6) }\end{array}$ \\
\hline
\end{tabular}


Continuación Cuadro No 2

\begin{tabular}{|l|l|l|}
\hline Tecnología & \multicolumn{1}{|c|}{$\begin{array}{c}\text { Categoría de } \\
\text { Análisis }\end{array}$} & $\begin{array}{l}\text { Perspectiva de los Migrantes por Estilo de vida de Pucón } \\
\text { de sus innovaciones socioculturales }\end{array}$ \\
\hline $\begin{array}{l}\text { De la vida } \\
\text { (Sabiduría) }\end{array}$ & $\begin{array}{l}\text { Obtención de } \\
\text { ganancias por } \\
\text { generar beneficios } \\
\text { sociales y } \\
\text { ambientales a la } \\
\text { sociedad }\end{array}$ & $\begin{array}{l}\text { "...La definición es preciosa, por eso es que maravilla a } \\
\text { mucha gente, porque en el fondo lo que tú dices es el } \\
\text { lucro como condición de hacerlo bien en la sociedad y } \\
\text { en el medio ambiente. No al revés, no es el lucro a costa } \\
\text { del medio ambiente y de la sociedad. Entonces si tú no } \\
\text { eres capaz de generar utilidades... entonces nosotros de- } \\
\text { cimos el problema no es el lucro, el problema es la mane- } \\
\text { ra de conseguir el lucro ¿me entiendes? Entonces de ahí } \\
\text { viene la segunda consigna que es no son las mejores em- } \\
\text { presas del mundo, son las mejores para el mundo" (UR4). }\end{array}$ \\
\hline
\end{tabular}

Fuente: Elaboración propia basado en De Souza et al. (2006).

\section{Consideraciones Finales}

Este trabajo ha buscado conocer las tecnologías socioculturales desplegadas por migrantes de amenidad y por estilo de vida en la ciudad de Pucón, e indagar en qué medida ellas contribuyen a un proceso de innovación sustentado en el intercambio cultural entre los migrantes y los habitantes de las comunidades receptoras desde la visión de los migrantes emprendedores.

Producto del análisis de las entrevistas realizadas y las consideraciones teóricas para abordar el problema se concluye que las tecnologías socioculturales para la innovación son producto de la emergencia de lo nuevo como acumulación histórica de horizontes y prácticas de los grupos involucrados, migrantes de amenidad/por estilos de vida y pobladores locales.

Por otra parte se advierte la importancia asignada por los migrantes entrevistados a la tecnología intelectual, es decir la tecnología de la interpretación, que maneja ideas, conceptos, teorías, paradigmas, metáforas y analogías para comprender la realidad a transformar. En este sentido la inserción en un nuevo contexto de vida, importa en muchos de los casos estudiados para disparar una transformación personal, que busca compartirse a través de diferentes proyectos. Las tecnologías sociales puestas en juego en ellos son la manifestación concreta de los cambios de visión de los sujetos de las formas de interpretar su visión del mundo y de sus relaciones con los demás y su entorno.

A partir de que los migrantes experimentan un cambio en su interpretación de cómo funciona la realidad y en el régimen de verdades tienen un comportamiento consciente y comprometido con ciertas prácticas. Entre ellas, la capacidad de escucha, se constituye en una tecnología social básica para la interacción y para el inicio del proceso de innovación.

Considerando que la migración por estilos de vida da lugar a interacciones sociales específicas y contingentes, y a procesos de articulación global-local, donde se pone en juego el capital cultural de los migrantes y sus trayectorias personales desarrollan tecnologías socioculturales 
críticas como la capacidad para detectar "anomalías" a las que se consagran y se constituyen en el motor de sus proyectos. Esta tecnología de vida da sentido a su existencia y a su relación con el contexto. De manera que en su hacer los migrantes por estilo de vida generan procesos de resignificación de las tecnologías de la sabiduría, intelectuales y sociales en su búsqueda de lograr sintonía con las realidades, necesidades y aspiraciones con los actores de la comunidad.

Estos procesos pueden ser interpretados como tecnologías políticas "en la medida que negocia criterios y valores para decidir sobre la inclusión y la exclusión, solidaridad y egoísmo, sensibilidad e indiferencia, regímenes democráticos y autoritarios, individualismo y colectivismo" (De Souza et al., 2006).

Las tecnologías socioculturales implícitas en las empresas sociales, llamadas empresas B en Chile desarrolladas por algunos de los migrantes por estilo de vida, plantean como innovación la generación de ganancias para ofrecer productos o servicios que promueven beneficios sociales y medioambientales a sus comunidades.

En síntesis, se aprecia que en las tecnologías socioculturales desplegadas por los migrantes de amenidad y por estilos de vida existe evidencia de la importancia de la consideración del modo contextual para comprender las formas que asumen los procesos de innovación, donde "el contexto es la referencia, la interacción la estrategia y la ética el garante de la sostenibilidad de todas las formas y modos de vida en el planeta" (De Souza Silva et al., 2007).

Los resultados de esta investigación muestran que los migrantes de amenidad y por estilos de vida que han hecho a la Ciudad de Pucón su lugar de residencia en los últimos años dan cuenta un importante potencial de transformación en términos de innovaciones sociales a partir del análisis de sus perspectivas. Estos resultados sugieren que los fenómenos de migración examinados revisten un notable interés para comprender las transformaciones económicas, sociales y culturales que se están evidenciando en numerosas localidades de Chile, especialmente en aquellas turísticas. Estas transformaciones deben ser adecuadamente interpretadas para dimensionar los efectos sobre el medio receptor y cautelar los beneficios y problemas que acarrean en la formulación e implementación de políticas territoriales para estos lugares, caracterizados como destino de migrantes por estilos de vida y de amenidad.

Asimismo, las categorías de análisis relativas a las dimensiones de las tecnologías socioculturales identificadas son un punto de partida para promover el estudio de procesos de innovación social en América Latina que permitan generar mejores condiciones de vida para la población y hacer un uso más adecuado de su patrimonio.

\section{Referencias bibliográficas}

BENSON, M. \& O'REILLY, K. Migration and the search for a better way of life: a critical exploration of lifestyle migration. The Sociological Review, 2009, No 57, p. 608-625.

BENSON, M. The British in rural France: Lifestyle migration and the ongoing quest for a better way of life. Manchester: Manchester University Press, 2011. 
BORSDORF, A. \& HIDALGO, R. Searching for Fresh Air, Tranquility and Rural Culture in the Mountains: A New Lifestyle for Chileans? Die Erde, 2009, Vol. 140, № 3, p. 275-292.

CHAVERRI, P. Cultural and environmental amenities in peri-urban change: the case of San Antonio de Escazú. In: MOSS, L.A.G. (Editor). The Amenity Migrants: Seeking and Sustaining Mountains and their Cultures. Cambridge: Wallingford Press, 2006, p. 187-199.

CÓRDOBA CEJUDO, R. Capacidades y libertad: una aproximación a la teoría de Amartya Sen. Revista Internacional de Sociología, 2007, Vol. LXV, No 47, p. 9 - 22.

DE SOUZA DA SILVA, J. El arte de cambiar a las personas que cambian las cosas. Conferencia presentada en el II Congreso Internacional de Formación Emprendedora", organizado por la Universidad Tecnológica América (UNITA), Quito, 2007.

DE SOUZA SILVA, J.; CHEAZ, J.; SANTAMARÍA, J.; MATO BODE, M.A.; VALLE LIMA, S.; GOMES DE CASTRO, A.M.; SALAZAR, L.; MAESTREY, A.; RODRÍGUEZ, N.; SAMBONINO, P. y ÁLVAREZ-GONZÁLEZ, F.J. La Innovación de la Innovación Institucional. De lo universal, mecánico y neutral a lo contextual, interactivo y ético desde una perspectiva latinoamericana. Quito: Red Nuevo Paradigma, 2007.

ESPINOZA, L.; ZEBRYTE, I. y ZUNINO, H. . Análisis del discurso de los migrantes de estilo de vida utópicos en la Patagonia andino lacustre. En: BORSDORF, A.; SÁNCHEZ, R.; HIDALGO, R. y ZUNINO, H.M. (Editores). Los riesgos traen oportunidades, Transformaciones globales en los Andes sudamericanos. Santiago de Chile: Serie GeoLibros, Instituto de Geografía- Pontificia Universidad Católica de Chile / Instituto de Geografía, Universidad de Innsbruck / Academia de Ciencias Austriaca, 2014, p. 263-274.

GONZÁLEZ, R.; OTERO, A.; NAKAYAMA, L. y MARIONI, S. Las movilidades del turismo y las migraciones de amenidad: problemáticas y contradicciones en el desarrollo de centros turísticos de montaña. Revista de Geografía Norte Grande, 2009, № 44, p. 75-92.

HAYES, M. Moving South: The Economic Motives and Structural Context of North America's Emigrants in Cuenca, Ecuador. Mobilities, 2014, Vol. 10, No 3, p. 267-284.

HIDALGO, R.; ZUNINO, H.; BENDER, O. \& BORSDORF, A. Metro-peripheral and lakeside amenity settlement: Chilean case studies. In: MOSS, L.A.G. \& GLORIOSO, R.S. (Editors). Global Amenity Migration: Transforming Rural Culture, Economy \& Landscape. Kaslo, BC \& Port Townsend, WA: New Ecology Press, 2014, p. 311-328.

INSTITUTO NACIONAL DE ESTADíSTICAS (INE). Censo de Población y Vivienda 2002. Santiago de Chile: INE, 2003.

JANOSCHKA, M. Imaginarios del turismo residencial en Costa Rica. Negociaciones de pertenencia y apropiación simbólica de espacios y lugares: una relación conflictiva. En: MAZÓN, T.; HUETE NIEVES, R. y MANTECÓN TERÁN, A. (Editores). Construir una nueva vida: los espacios del turismo y la migración residencial. Santander: Milrazones, 2011, p. 81-102. 
JANOSCHKA, M. Nuevas geografías migratorias en América Latina: prácticas de ciudadanía en un destino de turismo residencial. Scripta Nova Revista electrónica de Geografía y Ciencias Sociales, 2013, Vol. XVII, No 439. Disponible en Internet: http://www.ub.edu/geocrit/sn/sn439.htm

JARVIS, P. Learning to be a person in society: Learning to be me. New York: Routledge, 2009.

JARVIS, P. Learning from Everyday Life. HSSRP, 2012, Vol. I, Nº 1, p. 1-20.

MARACAIDA, B. y ZANFARDINI, M. Explorando la innovación social: conceptos de partida y desafíos para el turismo. Anuario de Estudios en Turismo - Investigación y Extensión, 2016, Vol. XI, p. $115-132$

MARGULIS, M.; URRESTI, M. y LEWIN, H. Intervenir en la Cultura. Más allá de las políticas culturales. Buenos Aires: Biblos, 2014.

MARCHANT, C. y ROJAS, F. Transformaciones locales y nuevas funcionalidades económicas vinculadas a las migraciones por amenidad en la Patagonia chilena. Journal of Alpine Research, 2015, Vol. 103, No 3. Disponible en Internet: http://rga.revues.org/2998

MATOSSIAN, B.; ZEBRYTE, I. y ZUNINO, H.M. Europeos y estadounidenses en la NorPatagonia andina: nuevas experiencias. Revista CIDOB d'Afers Internacionals, 2014, № 106-107, p. 237-259.

MCINTYRE, N. Rethinking Amenity Migration: Integrating Mobility, Lifestyle and Social-Ecological Systems. Die Erde, 2009, Vol. 140, No 3, p. 229-250.

MOSS, L.A.G. (Editor). The Amenity Migrants: Seeking and Sustaining Mountains and their Cultures. Cambridge: Wallingford Press, 2006, p.329.

MUNICIPALIDAD DE PUCÓN/UNIVERSIDAD DE LA FRONTERA. Plan de Desarrollo Comunal Pucón. 2010-2014. Pucón: Instituto de Desarrollo Local y Regional, 2010, p.19-20

NAJMANOVICH, D. Dinámica Vincular: territorios creados en el juego. Psicoanálisis de las Configuraciones Vinculares, Asociación Argentina de Psicología y Psicoterapia de Grupo, 2001, Vol. 24, No 2, p. 87-108.

OTERO, A.; NAKAYAMA, L.; MARIONI, S.; GALLEGO, E.; LONAC, A.; DIMITRIU, A.; GONZÁLEZ, R. \& HOSID, C. Amenity migration in the Patagonian mountain community of San Martín de los Andes, Neuquén, Argentina. In: MOSS, L.A.G. (Editor). The Amenity Migrants: Seeking and Sustaining Mountains and their Cultures. Cambridge: Wallingford Press, 2006, p. 200-213.

OTERO, A; GALLEGO, E. \& LONAC, A. Amenity Migration: Challenge for Better Understanding Gateway Communities and National Parks to Conserve Common Resources (Case Study of San Martín de los Andes and Lanín National Park). Ponencia presentada en Congreso Internacional "Understanding and Managing Amenity-led Migration in Mountain Regions (ALMMR)". Banff, 2008, p. 15.

OTERO, A. Urbanizaciones de Migración de Amenidad en el Corredor de Siete Lagos, Neuquén, Patagonia Argentina. Amenazas para un futuro cercano. Ponencia presentada en Foro Internacio- 
nal Saberes, Sabidurias e Imaginarios. Centro Canadiense de la Universidad Nacional de Córdoba, Córdoba, 2009, p.11.

OTERO, A. \& GONZÁLEZ, R. Amenity Migration. A Growing Shadow of Tourism: Contradictions in Local Development, Case Studies in Argentina. In: MOSS, L.A.G. \& GLORIOSO, R.S. (Editors). Amenity Driven Change: The New Global Countryside. Kaslo, BC \& Port Townsend, WA: New Ecology Press, 2014, p. 329-342.

OTERO, A. y GONZÁLEZ, R. Repensando el desarrollo de destinos turísticos: del valor centrado en el uso del suelo a la valorización de la creatividad para la innovación. Ponencia presentada en el VI Congreso Latinoamericano de Investigación Turística. Neuquén, 2014.

SARDINHA, J. Lifestyle Migrants in Central Portugal: Strategies of Settlement and Socialization. In: JANOSCHKA, M. \& HAAS, H. (Editors). Contested Spatialities, Lifestyle Migration and Residential Tourism. New York: Routledge, series on Contemporary Geographies of Leisure, Tourism and Mobility, 2013, p. 174-189.

SANCHEZ, R. Un lugar cerca de las estrellas. Turismo y migración de amenidades en el norte de Chile. BORSDORF, A.; SÁNCHEZ, R.; HIDALGO, R. y ZUNINO, H.M. (Editores). Los riesgos traen oportunidades, Transformaciones globales en los Andes sudamericanos. Santiago de Chile: Serie GeoLibros, Instituto de Geografía- Pontificia Universidad Católica de Chile / Instituto de Geografía, Universidad de Innsbruck / Academia de Ciencias Austriaca, 2014, p. 275-293.

THERRIEN, C. Quest migrants: French people in Morocco searching for elsewhereness. In: JANOSCHKA, M. \& HAAS, H. (Editors). Contested Spatialities, Lifestyle Migration and Residential Tourism. New York: Routledge, series on Contemporary Geographies of Leisure, Tourism and Mobility, 2013, p. 108-123.

THOMAS, H. Tecnologías para la inclusión social y políticas públicas en América Latina. Documento de Trabajo de la Red de Tecnologías para la Inclusión Social (redtisa.org), s/f. Disponible en Internet: http://www.redtisa.org/Hernan-Thomas-Tecnologias-para-la-inclusion-social-y-politicas-publicas-en-America-Latina.pdf contactado 16.06.15

VERGARA, L.; ROZAS, M. y ZUNINO, H.M. Los imaginarios urbanos y la arquitectura de Puerto Varas. Encrucijada entre lo local y lo global. AUS, 2013, Vol. 14, № 5. Disponible en Internet: http:// mingaonline.uach.cl/pdf/aus/n14/art05.pdf

ZEBRYTE, I.; MEDINA, V. y RITZ, K. Instancias sociales, formales de participación ciudadana para migrantes internacionales no convencionales en Chile. Revista Chilena de Derecho y Ciencias Políticas, 2015, Vol. 6, № 2, p. 117-143.

ZUNINO, H. y HIDALGO, R. En busca de la utopía verde: migrantes de amenidad en la comuna de Pucón, IX Región de la Araucanía, Chile. Scripta Nova Revista Electrónica de Geografía y Ciencias Sociales, 2010, Vol. XIV, No 331. Disponible en Internet: http://www.ub.edu/geocrit/sn/sn-331/sn331-75.htm 
ZUNINO, H.; HIDALGO, R. y MARQUARDT, E. Vivienda social y segregación espacial en la Ciudad de Pucón, Chile: entre el enclaustramiento y la integración con el hábitat turístico. Revista INVI, 2011, Vol. 26, Vol. 71, p. 15-55.

ZUNINO, H.; HIDALGO, R. \& ZEBRYTE, I. Utopian Lifestyle Migrants in Pucón, Chile: Innovating Social Life and Challenging Capitalism. In: JANOSCHKA, M. \& HAAS, H. (Editors). Contested Spatialities, Lifestyle Migration and Residential Tourism. New York: Routledge, series on Contemporary Geographies of Leisure, Tourism and Mobility, 2013, p. 113-125.

ZUNINO, H. \& ZEBRYTE, I. Pursuing the essence of existence: the daily quest of utopian migrants in the City of Pucón, Northern Patagonia. In: TORKINGTON, K.; DAVID, I. \& SARDINHA, J. (Editors). Practising the Good Life: Lifestyle Migration in Practices. London: Cambridge Scholars Publishing, 2015, p. 67-85.

ZUNINO, H.; ESPINOZA, L. y VALLEJOS, A. Movilidades contemporáneas y agentes de transformación en la NorPatagonia Chilena. Revista de Estudios Sociales, 2016, Vol. 55, No 1, p. 163-176. 
